FORMATION Formation emploi

Revue française de sciences sociales

143 | Juillet-Septembre 2018

Le retour en formation : une vraie chance?

\title{
La pédagogie, levier de professionnalisation pour les écoles de la deuxième chance?
}

Pedagogy as professionalization leverage in the schools of the "Second Chance program " from the Ardennes establishment: between innovation and resistance Die Pädagogik als Hebel zur Professionalisierung für Schulen der zweiten Chance La pedagogía, ¿palanca de profesionalización de la segunda oportunidad para las escuelas?

\section{Emmanuelle Leclercq et Laurent Béjot}

\section{(2) OpenEdition}

Journals

Édition électronique

URL : http://journals.openedition.org/formationemploi/6253

DOI : 10.4000/formationemploi.6253

ISSN : 2107-0946

Éditeur

La Documentation française

Édition imprimée

Date de publication : 20 novembre 2018

Pagination : 119-138

ISSN : 0759-6340

Référence électronique

Emmanuelle Leclercq et Laurent Béjot, «La pédagogie, levier de professionnalisation pour les écoles de la deuxième chance ? », Formation emploi [En ligne], 143 | Juillet-Septembre 2018, mis en ligne le 20 novembre 2020, consulté le 05 novembre 2020. URL : http://journals.openedition.org/ formationemploi/6253; DOI : https://doi.org/10.4000/formationemploi.6253 


\title{
La pédagogie, levier de professionnalisation pour les écoles de la deuxième chance?
}

\begin{abstract}
EMmANUELLE LeCLERCQ
Maître de conférences HDR (habilité à diriger des recherches), sociologue au Centre d'études et de recherches sur les emplois et les professionnalisations- CEREP (AE 4692)

LAURENT BÉJOT

Docteur en sociologie, chercheur associé au Centre d'études et de recherches sur les emplois et les professionnalisations- CEREP (AE 4692). Directeur-adjoint de l'Établissement public local d'enseignement et de formation professionnelle agricoles de Rethel, chargé de la direction du Centre de formation professionnelle et de promotion agricole (CFPPA)
\end{abstract}

Résumé

La pédagogie, levier de professionnalisation pour les écoles de la deuxième chance?

Cet article analyse le processus de professionnalisation inachevé, en œuvre dans les E2C (Écoles de la deuxième chance) de Champagne-Ardenne. L'analyse revient sur les leviers mis en place afin d'accompagner et d'harmoniser les pratiques des formateurs, tant d'un point de vue organisationnel que de celui de l'offre de formation continue et d'expérimentation pédagogique. Nous montrerons que l'évolution de l'organisation pédagogique par projet induit des nouvelles pratiques pour l'établissement et pour ses formateurs.

Mots clés : professionnalisation, formateur, pédagogie, accompagnement en formation, accompagnement professionnel, projet d'établissement, organisation du travail, activité professionnelle, évaluation, école de la deuxième chance

Abstract

Pedagogy as professionalization leverage in the schools of the « Second Chance program » from the Ardennes establishment: between innovation and resistance

This article analyses the unfinished profesionalization process in Champagne-Ardenne second chance schools (E2C). The analysis focuses on the reflexions and the actions to guide and standardize the trainer's practices. This means evolution in terms of work organization, training of trainers' and pedagogical testing. We argue that the evolu- 
tion of the organization of the pedagogical project leads to new practices for both the school and the trainers.

Keywords: professionalisation; trainer ; pedagogy ; coaching in training; coaching; school work plan; work organisation; vocational activity; evaluation; second-chance school

Journal of Economic Literature: J 68 ; J 44 ; M 54

Traduction : Auteur-e-s.

Le dispositif E2C (Écoles de la deuxième chance) fête ses vingt ans. Il a été créé en 1997, sur la base des principes inscrits dans le Livre Blanc "Enseigner et apprendre: vers la société cognitive " ${ }^{1}$. Cet article revient sur le processus de professionnalisation en cours dans le dispositif E2C en Champagne-Ardenne, l'encadrement et l'accompagnement des formateurs et le rôle de la pédagogie dans cette professionnalisation. Celle-ci est abordée selon trois niveaux d'analyse et leur interaction : la coordination régionale des $\mathrm{E} 2 \mathrm{C}$ Champagne-Ardenne, la direction de trois écoles de la deuxième chance attachées à un centre ardennais de formation professionnelle et de promotion agricole (CFPPA) et les formateurs de ces trois écoles. ${ }^{2}$

La coordination régionale de l'E2C Champagne-Ardenne est portée historiquement par le centre de formation interprofessionnel de Châlons-en-Champagne, aujourd'hui Centre Alméa. Il présente la particularité d'accueillir le siège du "Réseau des écoles de la $2^{\circ}$ chance France ". L'association de ce réseau, créée en 2004, inscrit dans sa charte la valorisation de la pédagogie active, par projet, l'alternance ${ }^{3}$. Le Centre de formation professionnelle et de promotion agricole (CFPPA) a ouvert sa première école en 2002 (site A). Il en ouvre une deuxième en 2009 (Site B), et enfin une troisième en 2013 (site C). Aujourd'hui les formateurs des trois écoles constituent une équipe de dix-neuf personnes, à savoir un responsable pédagogique, quatorze formateurs, quatre personnes en charge de la gestion administrative et financière.

1. Dans ce livre blanc, présenté par Édith Cresson et Padraig Flynn, la Commission européenne fixe cinq objectifs : encourager l'acquisition de nouvelles connaissances, rapprocher l'école de l'entreprise, lutter contre l'exclusion, maîtriser les trois langues communautaires, traiter sur un plan égal l'investissement physique et l'investissement en formation (prévoir une évolution du traitement fiscal et comptable des crédits destinés à la formation).

2. La Champagne-Ardenne, bassin fondateur du réseau E2C France, va imaginer un mode de gouvernance original et unique au niveau national, fondé sur un pilotage régional appuyé sur des établissements indépendants.

3. Le terme de pédagogie utilisé dans le texte se réfère à ces pédagogies et leurs formes d'accompagnement. 
Afin d'analyser les pratiques professionnelles contrastées des formateurs comme des institutions, nous mobiliserons les notions de professionnalisation et de professionnalité. La professionnalisation est considérée dans la littérature qui lui est consacrée, notamment en sociologie et en sciences de l'éducation, comme un processus devant améliorer les pratiques et l'engagement professionnel (Demazière, 2009). Pour renforcer la compréhension des processus de professionnalisation, nous mobiliserons également, tout au long du texte, la notion de professionnalité singulière dans cette activité d'accompagnement de jeunes. Nous nous appuierons sur la définition de la professionnalité fournie par François Aballéa, qui croise l'expertise et la déontologie dans l'action (Aballéa, 1992). Dans ces processus, nous analyserons quelles sont les améliorations mises en place, mais aussi les limites identifiées.

Dans une première partie, nous montrerons comment la professionnalisation du dispositif se met en place afin de renforcer les finalités d'accompagnement des jeunes bénéficiaires vers une sortie positive. Cette professionnalisation impacte profondément la manière d'exercer l'activité de formateurs, non sans certaines formes de résistance.

Nous reviendrons, dans une deuxième partie, sur la manière dont l'établissement ardennais conduit la professionnalisation autour de l'activité dédiée à la préparation de cette sortie positive, par la construction du projet des stagiaires (Techniques de recherche d'emploi - TRE) centrée sur des pédagogies d'orientation. Cette réflexion se trouve confrontée à l'hétérogénéité des profils des formateurs et de leurs pratiques. Nous analyserons comment, grâce à la formation continue, l'institution guide l'harmonisation des pratiques d'accompagnement, et quel usage en font les formateurs.

Nous aborderons, dans une troisième partie, ce processus de professionnalisation relatif aux pédagogies par projet et l'approche par compétences. Nous montrerons que les expérimentations construites en matière pédagogique sont freinées par les injonctions d'évaluation du dispositif E2C et ne sont pas sans effet sur les pratiques des formateurs et leur professionnalité. 


\section{Encadré 1. Méthodologies de la recherche}

La recherche présentée a été réalisée en 2016 et 2017, grâce au croisement de plusieurs sources et données. Ont été analysés des donnés de cadrage régional des résultats produits par la coordination régionale des E2C (Écoles de la deuxième chance), des données sociales de l'établissement ardennais (parcours des formateurs et leur participation à la formation continue), ainsi que des documents d'évaluation des stagiaires et leur suivi sur la plateforme «Cyclise ». $\left({ }^{*}\right)$

Des observations ont été réalisées. Ont ainsi observées deux réunions de la coordination régionale regroupant toutes les E2C de la Région Champagne-Ardenne. Elles abordaient les thématiques pédagogiques de fonctionnement de certains outils de la plateforme collaborative. Des observations ont été menées sur le site $A$ d'un comité de pilotage de cette école

$\left.{ }^{* *}\right)$ et deux observations lors de séances de travail des formateurs avec les stagiaires sur la recherche d'emploi, sur les sites $A, B$ et $C$.

Des entretiens ont été conduits tout au long de la recherche : un entretien avec le coordinateur régional a été complété par trois entretiens avec le directeur du Centre de formation professionnelle et de promotion agricoles (CFPPA), trois entretiens avec la coordinatrice de l'établissement ardennais, à différents moments ; deux entretiens, l'un avec le chargé de mission en charge de l'évaluation des pratiques pédagogiques sur les techniques de recherche d'emploi, l'autre avec la chargée de mission chargée des questions pédagogiques de l'établissement ardennais. Des entretiens semi-directifs d'une à deux heures avec les équipes des formateurs et coordinateurs des trois sites ont été menés fin 2016 et début 2017 (trois personnes par site, sur un effectif de quatre ou cinq personnes selon les sites), soit sur l'ensemble du dispositif, quatorze personnes rencontrées, dont neuf formateurs. Les grilles d'entretien avec les formateurs et animateurs axent le questionnement sur le parcours et l'expérience de la personne interviewée, les approches et les outils utilisés, la vision du dispositif, le rapport à l'établissement et à la coordination régionale. Le traitement du discours est réalisé par une analyse de contenu thématique (Bardin, 1977).

$\left.{ }^{*}\right)$ : La plateforme Cyclise permet le suivi individuel des activités, et notamment l'alternance en entreprise, des stagiaires. Elle permet de comprendre la progression des stagiaires pour la remise à niveau. Le rôle de cette plateforme sera abordé en partie 3.

$\left.{ }^{* *}\right)$ : Le comité de pilotage se réunit en moyenne deux fois par an, avec une représentation des institutions régionales: Conseil départemental, Mairies, Communauté de commune, Mission insertion et développement social (MIDS), Conseil économique, social et environnemental régional (CESER), Foyer des jeunes et d'éducation populaire (FJEP), Cap Emploi, Pôle Emploi, Société nationale des Chemins de fer français (SNCF). 


\section{Réorganiser pour professionnaliser}

Richard Wittorski identifie comme niveaux de la professionnalisation, l'organisation du travail, les sujets et les activités (Wittorski, 2007). Si la professionnalisation est considérée comme une catégorie d'analyse (Demazière, op. cit.), nous aborderons, dans une première partie, la nécessité, portée par l'institution, de professionnaliser l'ensemble de l'organisation des activités professionnelles dédiées à l'accompagnement des stagiaires afin d'harmoniser et d'encadrer les pratiques pédagogiques des formateurs. Cette professionnalisation est menée de manière institutionnelle par une réflexion sur le contour de l'activité professionnelle et le recrutement des formateurs. Nous considérons, tout au long de l'article, l'activité professionnelle comme un processus de recomposition du travail au regard d'environnements organisationnels en perpétuelle évolution (Demazière, 2008).

Nous reviendrons, dans cette partie, sur la professionnalisation du dispositif par une réaffirmation des missions des écoles de la deuxième chance, une réorganisation du périmètre d'activité des formateurs et une attention accordée au recrutement de ces derniers.

\subsection{La professionnalité des formateurs : de l'aide sociale à l'accompagnement social}

Les E2C sont organisées généralement en écoles indépendantes. Mais sur le territoire champardennais, le principe d'un réseau regroupant une coordination " régionale » est singulier. Ainsi, sur ce territoire, la coordination anime ainsi le réseau en proposant un maillage régional alimenté par de nombreuses réunions de partage d'expérience, de mise en commun, de formation. Si elle réitère les objectifs de la charte des E2C, elle met néanmoins des moyens de progrès en place par une réflexion sur le recrutement des formateurs et la formation continue.

La coordination régionale est garante du label E2C pour l'ensemble des écoles du territoire champardennais Ce label induit une pédagogie axée sur l'alternance, la construction de projet d'insertion et l'autonomie des stagiaires. Au regard de l'histoire de l'évolution des E2C au sein de l'établissement ardennais, cela aboutit à la mise en place d'un axe de réflexion sur l'accompagnement des stagiaires et sur les évolutions de la professionnalité des formateurs.

En 2015, alors même que des difficultés importantes apparaissent sur les sites - pas assez de stagiaires, pas assez de sorties positives - le dispositif E2C de l'établissement se trouve confronté au décès de sa coordinatrice historique. Une nouvelle coordinatrice prend le relais dans un contexte de changement et de crise : évolution du modèle pédagogique, prévalence de la culture du résultat, accentuation des contrôles de tous ordres (du financier au pédagogique) imposés par la coordination régionale. Cette 
coordonnatrice, possédant une expérience d'une quinzaine d'années en formation, a pour mission d'engager une politique d'harmonisation des pratiques et des procédures.

Pour l'établissement, son arrivée va être l'occasion d'un recentrage sur la charte des E2C orientée vers la sortie en formation ou l'emploi. S'opère aussi une redéfinition de l'accompagnement. Les approches pédagogiques axées sur du rattrapage scolaire et de l'accompagnement social psychoaffectif se transforment en démarches actives faisant de l'apprenant l'acteur de son parcours (Astier, 2009). L'ancienne coordinatrice faisait prévaloir la dimension sociale sur le pilotage général : "On a gardé un jeune pendant deux ans et on ne lui a pas trouvé... ; il ne voulait pas aller en formation; il fallait qu'on arrête" (Formateur 14).

Désormais, la coordination et la direction du CFPPA rappellent constamment que le rôle des E2C est certes de rester à l'écoute des situations sociales, mais surtout de réagir en accompagnant les stagiaires vers les partenaires sociaux adéquats : "On se retrouvait avec des jeunes qui faisaient 2 semaines d'entreprise sur 6 mois ... on estimait qu'ils n'étaient pas prêts socialement; dans ce cas, on n'est pas le dispositif adapté" (Formateur 3). Cette réorganisation se heurte à une résistance des formateurs historiques, qui va se concrétiser par des départs au regard d'une équipe jusqu'alors stable (cinq départs et deux non renouvellements de contrat en raison d'une baisse du financement). Les départs concernent des formateurs favorisant une approche sociale. Ils voient en l'arrivée de la nouvelle coordinatrice des nouvelles façons de travailler jugés incompatibles avec leur professionnalité (Denecheau, Houdeville, Mazaud, 2015, p. 255).

Le recentrage sur la charte E2C entraîne une évolution de la professionnalité des formateurs. Il impacte également le processus de professionnalisation mis en œuvre par l'établissement ardennais.

\subsection{Une restructuration des pôles d'activité des formateurs}

L'analyse des entretiens et les observations de réunions de concertation mettent en avant, de manière récurrente chez les encadrants du dispositif, tant au niveau de la coordination que de l'établissement, que les résultats de sorties des stagiaires sont inégaux selon les sites et surtout ne sont pas bons. Les équipes encadrantes ont un discours commun relevant de la charte des E2C sur le lien entre pédagogies adaptées et succès dans le dispositif. Sur les sites champardennais, la moyenne régionale de sorties " positives ${ }^{4}$ des stagiaires est, en 2016 , de $48 \%$. Elle traduit partiellement la réalité et les

4. Une sortie positive, en 2016, signifie qu'un stagiaire est sorti pour poursuivre son parcours en formation qualifiante ou diplômante dans le cadre de la formation des adultes et/ou signe un contrat de travail (en alternance ou non) via des emplois d'une durée de plus de deux mois, en intérim ou encore vers des emplois aidés. 
types de difficultés de ces publics. En effet, les informations de suivi ne renseignent pas le suivi des jeunes qui adhèrent et sont acceptés dans le dispositif.

Ces résultats croisent des réalités économiques très contrastées de l'emploi et d'offres de formations post-dispositif et des profils sociaux des stagiaires qui diffèrent selon les territoires. Pour l'établissement ardennais, le site A, en partie intégré au CFPPA, bénéficie de ses réseaux et de sa culture, le site B favorise le travail pédagogique, sur le site C, l'offre de formation est moindre dans cette partie du nord des Ardennes et impose une mobilité vers Charleville.

Tableau 1 : Résultats des sorties des stagiaires

\begin{tabular}{|l|c|c|c|c|}
\hline \multicolumn{1}{|c|}{ Sorties } & Site A & Site B & Site C & Ensemble \\
\hline Sorties positives & & & & \\
Dont sorties vers formations & $51 \%$ & $43 \%$ & $26 \%$ & $40 \%$ \\
& $11 \%$ & $14 \%$ & $2 \%$ & $9 \%$ \\
\hline Abandons durant les 6 premières semaines & $33 \%$ & $34 \%$ & $22 \%$ & $30 \%$ \\
\hline Orientation vers dispositif d'insertion & $14 \%$ & $15 \%$ & $25 \%$ & $18 \%$ \\
\hline Autres sorties non maitrisables & $2 \%$ & $8 \%$ & $27 \%$ & $12 \%$ \\
\hline Moyenne & $100 \%$ & $100 \%$ & $100 \%$ & $100 \%$ \\
\hline
\end{tabular}

Source : données du bilan d'activité établissement année 2016.

Cependant, ni la coordination régionale, ni l'établissement ardennais ne sont en mesure, malgré de nombreuses données disponibles issues des audits et du suivi des stagiaires, d'apporter une réponse plus argumentée sur l'impact des pédagogies mises en place au regard de la spécificité des publics. Toutefois, comme le souligne TiberghienBovy (2007), il est délicat de généraliser des positions ou des éléments explicatifs sur les pratiques pédagogiques engagées dans des dispositifs pour des publics de faible niveau de qualification. En effet, le contexte est marqué par la fluctuation des politiques de formation destinées à ce public, les approches multiples des formateurs et la diversité des difficultés rencontrées par ces publics, pour lesquels, en outre, la représentation du monde du travail peut être multiple (Fourdrignier, 2004, Céreq, 2003).

La coordination et les établissements poursuivent leur réflexion sur l'accompagnent des stagiaires, l'étendue des activités professionnelles des formateurs, souvent floues et interconnectées (Leclercq, 2005) : "Des fois, on ne sait plus ce que l'on doit faire et ce quion ne doit pas faire" (Formateur 6, H). Un séminaire commun aux E2C champardennaises est alors organisé par la coordination, en 2014. Trois pôles d'activités sont identifiés par les représentants des écoles :

- "pôle formation " : remise à niveau des stagiaires. Profil formateur : niveau $\mathrm{Bac}+3$ recommandé ;

- "pôle entreprise ": construction du projet du stagiaire (Techniques de Recherche d'Emploi-TRE). Profil formateur : Bac +3 recommandé + expérience professionnelle ; 
- "pôle animation » socioculturelle : accompagnement des jeunes sur des projets ou des sorties. Profil formateur : diplôme spécialisé + expérience professionnelle.

Bien que ces données sur les parcours des bénéficiaires soient régulièrement communiquées dans le dispositif, elles restent difficiles à analyser dans le lien entre pratiques pédagogiques et résultats de sorties. Dans le discours de la coordination régionale et de l'établissent ardennais, leur connaissance devrait contribuer à l'amélioration du dispositif et à la professionnalisation des formateurs avec une redéfinition, dans les trois pôles, du périmètre de l'activité des formateurs, collectivement discutée.

\subsection{Une logique de recrutement}

Cette professionnalisation fondée sur la précision et la complexification des activités s'est accompagnée d'une réflexion sur les recrutements des équipes.

Selon la philosophie de départ des E2C et les consignes de la coordination, le recrutement pour l'école A s'est réalisé jusqu'à l'ouverture de la dernier école (site C), sur des profils d'animateurs, privilégiant l'animation et l'encadrement socioculturel des publics. En termes de diplôme, ces personnes pouvaient avoir un profil d'animateur jeunesse avec un BP JEPS (Brevet professionnel de la jeunesse de l'éducation populaire et du sport, spécialité animation sociale). Ces profils, à la suite du séminaire de 2014 animé par la coordination, se complexifient avec une définition élargie de l'animation.

En effet, la définition du pôle 3 Animation induit une animation de projet qui dépasse la seule considération de sorties socioculturelles. Comme le précise le Directeur de l'établissement ardennais, "la frontière entre l'animateur et le formateur s'est vraiment estompée. On ne savait pas bien faire la différence, donc on n'en a quasiment plus fait".

Parallèlement, cette répartition en trois pôles entraîne de nouveaux recrutements afin de renforcer le pôle 2 Entreprise, levier du dispositif (Legal, 2015). En raison du rattachement de l'E2C à un centre de formation professionnelle et du profil de la nouvelle coordinatrice, des formateurs issus du monde de la formation sont recrutés. Beaucoup d'entre eux arrivent sur le dispositif E2C après avoir eu une expérience de formateurs plus ou moins longue au CFPPA, et en ont intégré l'esprit et les manières de travailler. Même si le groupe des formateurs est très composite et confirme la littérature sur leur hétérogénéité (De Lescure, 2005 ; Leclercq, op. cit.), il n’en reste pas moins que le passage par l'établissement ardennais est un facteur important de leur socialisation et de professionnalité.

Si peu d'éléments objectifs sont aujourd'hui avancés pour analyser les résultats pédagogiques de l'accompagnement des stagiaires, la professionnalisation de l'organisation de l'ensemble des E2C s'est bien mise en place. Elle se lit dans une capacité à se réorganiser, à réfléchir en commun, à repenser les limites de l'activité professionnelle des formateurs et dans ses recrutements. 


\section{Professionnaliser le suivi du projet d'insertion des stagiaires}

Raymond Bourdoncle (2000) met en avant le fait que la professionnalisation concerne tout autant le domaine de l'activité professionnelle que le groupe qui la réalise, les savoirs, les personnes, la formation. Nous analyserons, dans cette deuxième partie, la manière dont les formateurs construisent et animent le travail de recherche d'emploi (TRE), renvoyant aux imbrications des dimensions évoquées par cet auteur. Nous reviendrons également sur l'usages par les formateurs de la formation continue, illustrant la professionnalité de ces derniers.

\subsection{Des profils de formateurs disparates}

L'établissement ardennais, dans l'objectif d'améliorer ses taux de sorties positives, opte pour une stratégie d'harmonisation et d'expérimentation pédagogiques sur l'ensemble des trois sites. En 2015, il entreprend alors de faire réaliser un bilan des pratiques des formateurs sur le travail de recherche d'emploi (TRE), par un formateur du CFPPA possédant une expérience dans le domaine de la pédagogie d'orientation et d'insertion. Dans l'établissement, les niveaux, les spécialités de diplômes et les expériences sont très disparates (De Lescure, op. cit. ; Leclercq, op. cit.). Les niveaux de formation s'étendent du titre de formateur jusqu'au au niveau bac +5 , mais parfois avec des spécialités éloignées des pratiques pédagogiques. Certains sont titulaires de diplômes de l'insertion sociale, mais sans approche pédagogique. D'autres qui ont un titre de formateur, possèdent des expériences rarement en lien avec la formation. C'est également vrai pour les formateurs titulaires d'un titre de conseiller en insertion. Ce qui peut conduire à des lacunes pédagogiques non totalement comblées par l'organisation de la formation (voir partie 2.3). 
Tableau 2. Les parcours et expériences des formateurs

\begin{tabular}{|c|c|c|c|c|c|c|c|c|c|}
\hline$\stackrel{\circ}{z}$ & 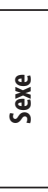 & ڤั̆ & gั & 总 紊 & $\frac{\text { 뜨 }}{\text { 음 }}$ & 包 흘 & 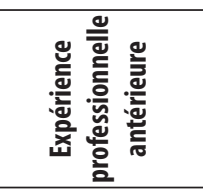 & 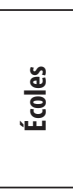 & 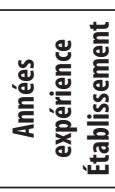 \\
\hline 1 & $\mathrm{~F}$ & Responsable & 46 & 2 & Licence & $\begin{array}{l}\text { Physique } \\
\text { chimie }\end{array}$ & Formatrice conseiller & Toutes & 1,5 \\
\hline 2 & $\mathrm{H}$ & Formateur & 39 & 3 & Titre & $\begin{array}{c}\text { Formateur } \\
\text { d'adultes }\end{array}$ & $\begin{array}{l}\text { Chargé mission ingé- } \\
\text { nierie sociale }\end{array}$ & \multirow[t]{6}{*}{ Site A } & 1 \\
\hline 3 & $F$ & Formateur & 48 & 3 & Titre & $\begin{array}{l}\text { Formation } \\
\text { d'adultes }\end{array}$ & $\begin{array}{c}\text { Responsable de } \\
\text { magasin }\end{array}$ & & 2 \\
\hline 4 & $F$ & Formateur & 32 & 2 & $\begin{array}{c}\text { Licence } \\
\text { Professionnelle }\end{array}$ & $\begin{array}{l}\text { Métier de } \\
\text { l'insertion }\end{array}$ & $\begin{array}{c}\text { Animatrice centre } \\
\text { loisirs }\end{array}$ & & 7,5 \\
\hline 5 & $\mathrm{H}$ & Formateur & 38 & 4 & Brevet d'Etat & $\begin{array}{l}\text { Educateur } \\
\text { sportif }\end{array}$ & Éducateur sportif & & 1,5 \\
\hline 6 & $\mathrm{H}$ & Formateur & 31 & 1 & Titre & $\begin{array}{l}\text { Responsable } \\
\text { commercial }\end{array}$ & Gérant d'entreprise & & 1 \\
\hline 7 & $\mathrm{~F}$ & Formateur & 27 & 1 & Master2 & Psychologie & Formatrice/conseiller & & 0,5 \\
\hline 8 & $\mathrm{H}$ & Formateur & 34 & 1 & Master 2 & $\begin{array}{l}\text { Sciences } \\
\text { Humaines }\end{array}$ & Professeur de collège & \multirow[t]{5}{*}{ Site B } & 1,5 \\
\hline 9 & $\mathrm{H}$ & Formateur & 32 & 4 & BP JEPS (*) & $\begin{array}{l}\text { Activités spor- } \\
\text { tives pour tous }\end{array}$ & Éducateur sportif & & 5,5 \\
\hline 10 & $\mathrm{~F}$ & $\begin{array}{c}\text { Formateur et } \\
\text { chargée de } \\
\text { mission }\end{array}$ & 36 & 1 & Master2 & $\begin{array}{l}\text { Sciences de } \\
\text { l'éducation }\end{array}$ & $\begin{array}{c}\text { Formatrice CFPPA } \\
\qquad(* *)\end{array}$ & & 2,5 \\
\hline 11 & $\mathrm{H}$ & Formateur & 47 & 2 & Deug & $\begin{array}{l}\text { Economie } \\
\text { gestion }\end{array}$ & $\begin{array}{l}\text { Formateur } \\
\text { informatique }\end{array}$ & & 12 \\
\hline 12 & $\mathrm{H}$ & Formateur & 30 & 2 & $\mathrm{LP}$ & $\begin{array}{l}\text { Métiers de } \\
\text { l'insertion }\end{array}$ & $\begin{array}{l}\text { Accompagnateur } \\
\text { social } \\
\end{array}$ & & 7,5 \\
\hline 13 & $F$ & Formateur & 33 & 3 & Titre & $\begin{array}{l}\text { Conseiller en } \\
\text { insertion }\end{array}$ & $\begin{array}{l}\text { Chargée d'accueil } \\
\text { mairie }\end{array}$ & \multirow[t]{3}{*}{ Site C } & 1,5 \\
\hline 14 & $H$ & Formateur & 35 & 2 & Licence & Géographie & $\begin{array}{l}\text { Animateur centre } \\
\text { loisirs }\end{array}$ & & 2,5 \\
\hline 15 & $\mathrm{H}$ & Formateur & 44 & 3 & Titre & $\begin{array}{c}\text { Conseiller } \\
\text { en insertion } \\
\text { professionnelle }\end{array}$ & $\begin{array}{l}\text { Responsable } \\
\text { administratif }\end{array}$ & & 5 \\
\hline
\end{tabular}

$\left.{ }^{*}\right)$ : Brevet professionnel de la Jeunesse, de l'Éducation populaire et du Sport. $\left(^{* *}\right)$ : Centre de formation professionnelle et de promotion agricoles. Source : données sociales de l'établissement - service administration des personnels.

\subsection{Des savoirs et des pratiques professionnelles multiples}

Les savoirs et les pratiques pédagogiques sont mobilisés dans les trois pôles précédemment décrits - formation, entreprise, animation. Le pôle entreprise est devenu le cour du dispositif, car le travail sur le projet professionnel, appelé " techniques de recherche d'emploi " (TRE), apparaît être la clé du « raccrochage » du stagiaire. Il fonde la notion de prise sur et avec le stagiaire, que d'autres auteurs ont bien mise en évidence dans la relation du travail social d'accompagnement de publics en retour vers l'emploi (Brun, Corteel, Pelisse, 2012). Il se réalise avec un formateur référent, originalité du dispositif E2C. 
Le bilan réalisé par l'établissement ardennais confirme des pratiques des formateurs multiples et peu harmonisées : "Premier constat, c'est que chacun a tendance à travailler dans son coin dans le sens où il n'y avait pas forcément mutualisation des différents outils qui existaient" (formateur 2). Il souligne le fait que les formateurs restent sur leurs acquis et les échanges d'expériences se réalisent bien souvent de manière informelle et sans méthode précise : "J'ai discuté avec X pour savoir comment elle faisait pour ses simulations d'entretiens, elle m'a répondu qu'elle n'en faisait jamais car elle n'aimait pas l'exercice... c'est pourtant assez essentiel!" (Formateur 4). Les formateurs ont tendance à mobiliser des outils existants, à les adapter à leurs besoins et à leur public: "Nous on a nos propres outils quion s'est construits, moi dans mes pratiques j'utile beaucoup l'outil multimédia" (Formateur 11). Ces outils relèvent de la pédagogique d'orientation. Des publications d'éditeurs proches de l'activation du développement vocationnel et professionnel (ADVP) ${ }^{5}$, comme "Chemin faisant " «Éditions qui plus est », sont très utilisées par les formateurs. Cependant, certains outils restent trop peu maîtrisés pour permettre aux formateurs d'aborder la démarche de construction de projet : "l'ADVP je connais, j'en ai entendu parlé, mais je n'ai pas suivi de formation" (Formateur 3).

Au final, pour l'établissement ardennais, on recense 286 outils utilisés par les formateurs et animateurs, regroupant 19 thématiques ${ }^{6}$. Les plus mobilisés sont les outils de validation d'étape dans les six semaines de positionnement, c'est-à-dire un suivi administratif du parcours du stagiaire. La multiplication des éléments, écrits, dossiers administratifs, rend le processus très lourd. En revanche, le suivi de la construction et de la validation du projet semble très peu outillé. De plus, la recherche d'emploi prime clairement sur la recherche de formation et est fortement centrée sur le stage.

Pour l'établissement ardennais, l'hétérogénéité des parcours des formateurs et des animateurs peut expliquer le fait que les attendus pédagogiques en matière de travail sur le projet ne semblent pas toujours maîtrisés : "Il n’y pas la connaissance théorique et pratique de base pour savoir ce qui est attendu... alors après, forcément, chacun voulant faire pour le mieux met en place des outils plus ou moins adaptés" (formateur 2). La méconnaissance des outils existants est réelle, ainsi que la difficulté d'harmoniser des pratiques.

5. L'activation du développement vocationnel et professionnel (ADVP) est une méthode anglo-saxonne, développée à partir des travaux de Carl Rogers (1968) et de Donald Winnicott (1975). Elle renvoie à "l'éducation des choix " par un travail d'exploration des métiers, un tri d'informations afin d'orienter les choix et entreprendre les démarches nécessaires. Elle s'est largement développée et diffusée, en France, grâce à des chercheurs de Lyon 2, et notamment à Geneviève Latreille (Leclercq, 2012).

6. Thématique des outils utilisés par les formateurs : Suivi parcours administratif (41 outils), Cohésion de groupe (6 outils), Connaissance de soi (35 outils), Élaboration de projet (12 outils), Environnement de travail recherché (5 outils), Connaissance des métiers (26 outils), Curriculum vitae (11 outils), Lettre de motivation (19 outils), Stage (36 outils), Développement comportemental (14 outils), Connaissance de l'entreprise (19 outils), Validation du projet (2 outils), Entretien d'embauche (31 outils), Recherche d'emploi (24 outils), Recherche de formation (3 outils), Portefeuille compétences (2 outils). 


\subsection{La formation comme moyen imparfait de professionnalisation}

Sont proposées aux formateurs 25 actions de formations, d'un à cinq jours, sur l'illettrisme, le projet professionnel. Elles se découpent en 27 sessions pour renforcer la compétence des formateurs autour des questions de pédagogie, de transmission, d'évaluation, et 32 sessions sur le projet professionnel et l'entreprise. L'établissement ardennais a souhaité accentuer le processus de professionnalisation en s'appuyant sur l'offre de formation de la coordination et sur un complément organisé à son initiative. Mais les appropriations de la formation diffèrent selon les bénéficiaires :

- Niveaux de diplômes : comme l'a bien démontré Claude Dubar (2015) notamment, ce sont les salariés possédant un haut niveau de diplôme qui se forment le plus. Les formateurs de niveaux licence et master de l'établissement ardennais représentent $60 \%$ de l'effectif formé au cours des deux années 2015 et 2016, et ont suivi $80 \%$ des heures de formation. Les $40 \%$ qui restent ont suivi $20 \%$ des heures de formation.

- Offre de formation : les formateurs se forment davantage sur les thématiques du "pôle formation" (60\%) que sur celles du Pôle entreprises (40\%). La mise en commun est plus aisée sur les thématiques de remise à niveau des connaissances que sur celles concernant le projet personnel.

- Pôle entreprises : les formations sur le TRE sont plus axées sur la difficulté des publics que sur une pédagogie d'orientation. La demande de formation au regard du peu d'expérience de certains formateurs s'oriente davantage sur la relation aux stagiaires que sur les contenus techniques et théoriques du TRE.

Lécueil de cette intention de professionnaliser n'est pas d'avoir mis un dispositif en place sans former (Duvoux, 2009), bien au contraire, puisqu'il y a pléthore de formations. Mais ces formations répondent partiellement à des questions individuelles sur l'accompagnement individualisé des stagiaires et les problèmes qu'il suscite, les pratiques pédagogiques personnelles, sans réelle mise en synergie.

\subsection{La préoccupation des formateurs est de maintenir du lien}

François Aballéa (1992) souligne la dimension déontologique dans la définition de la professionnalité : "J'appelle professionnalité, et j'attribue cette professionnalité à un individu et à un groupe, une expertise complexe et composite, encadrée par un système de références, valeurs et normes, de mise en cuvre, ou pour parler plus simplement, un savoir et une déontologie, sinon une science et une conscience" (op. cit., p. 47).

Dans le travail sur le TRE, cette dimension se lit dans la relation au jeune, qui semble essentielle et qui fonde la professionnalité des formateurs. Les nombreux entretiens individuels, durant la période où le stagiaire est sur les sites, sont des outils de travail où chacun gère cette situation de face-à-face avec sa sensibilité et son expérience : "J'attache 
beaucoup d'importance à l'entretien individuel avec le jeune pour connaitre l'historique et les centres d'intérêt» (Formateur 13).

On retrouve l'importance de cette relation de confiance tissée au fil du temps dans de nombreux discours des formateur TRE : "Ce n'est pas l'outil qui va permettre de travailler de façon satisfaisante, c'est plus la relation que l'on met en place entre le formateur et le jeune qui va nous permettre d'avancer dans le parcours" (Formateur 11).

Les entretiens et les observations que nous avons menés sur sites confirment l'importance d'un suivi qui finalement apparaît très individualisé, en lien direct avec le système du formateur référent. Ce qui engendre une forte responsabilité individuelle du formateur face à un échec non exprimé ni assumé de manière collective : "Le plus difficile dans mon métier, c'est l'échec avec un jeune qui abandonne " (Formateur 12). L'abandon est considéré comme échec, alors même que si un jeune n’est pas arrivé au bout du parcours, de nombreux exemples montrent qu'il a pu rebondir et trouver un emploi quelques temps après sa sortie du système.

La conception pédagogique du TRE repose sur les capacités de réflexivité et d'action du stagiaire. Or, les formateurs travaillant sur le TRE sont confrontés au problème d'un projet, existant ou non chez le stagiaire, susceptible de provoquer la motivation. Les observations sur sites révèlent la difficulté des jeunes à exprimer un projet et des types d'attachement ou de comportements relationnels que les formateurs doivent savoir décrypter (Blanchard \& Sontag, 2015, p. 143).

La prédominance d'un suivi individualisé, l'importante accordée aux entretiens individuels marquent les pratiques du formateur qui s’appuie sur son expertise, sa déontologie. Si ce mode de fonctionnement renforce le sentiment de responsabilité en cas d'abandon ou de non-placement, il diminue l'appropriation collective des outils.

\section{2.approche par projet et la professionnalisation collective des formateurs}

Didier Demazière (2009) souligne la dimension d'accumulation d'expériences qui permet d'améliorer la pratique professionnelle, tout en considérant le cadre limitatif et prescriptif de l'organisation du travail par des modèles managériaux impliquant des modalités de contrôle. Nous mettrons en avant, dans cette partie, les effets de cette injonction paradoxale de l'organisation et de ses modes de contrôle sur la professionnalisation et la professionnalité des formateurs. La volonté d'améliorer le travail pédagogique, affichée par la coordination régionale et l'établissement ardennais, vers un suivi des apprentissages davantage porté par le collectif, entraîne des évolutions pédagogiques importantes, sur lesquelles nous reviendrons. Nous montrerons qu'elles sont pourtant 
en contradiction avec les attendus de résultats s'agissant des taux de placement des jeunes bénéficiaires, mettant une pression supplémentaire sur les formateurs.

\subsection{La généralisation d'une pédagogie par projet est freinée par les référentiels}

Pour la coordination et l'établissement ardennais, faire évoluer les pédagogies en ouvre passe, notamment, par les pédagogies par projet et par compétences, malgré les nombreux freins. La base de données Cyclise, portée par la coordination depuis 2008, recense tous les stagiaires E2C. Elle permet en outre un suivi administratif et pédagogique de leur parcours en centre et en entreprise. Le fonctionnement actuel des E2C, au niveau de la coordination et de l'établissement ardennais, est fondé sur l'idée développée dans les années 1990 de la nécessité d'un socle de connaissances minimum (Tiberghien-Bovy, op. cit., p 19). La plateforme Cyclise est construite sur cette idée avec l'objectif d'une remise à niveau et une progression pédagogique disciplinaire (français, mathématiques, informatique).

Après les tests de niveau, des séquences pédagogiques sont proposées aux stagiaires. Cette ingénierie est fortement structurée, mais à partir de là, les formateurs recomposent leur accompagnement : "On a des séquences-type, mais j’essaie de raccrocher à leurs préoccupations et d'adapter" (Formateur 14). Ils mettent ainsi en œuvre ce que Laurent Riot (2010) appelle un style socio-éducatif souple » (Riot, ) s'adaptant aux publics dans le principe de l'individualisation du parcours.

Si le suivi du parcours de formation est très formalisé, les moments en entreprise sont peu valorisés et peu traduits en termes de compétences acquises, dans les comptes rendus établis par les formateurs. La plateforme Cyclise permet certes de suivre factuellement et linéairement les stagiaires dans le processus d'apprentissage et d'alternance, mais elle reste limitée pour formaliser les retours sur expériences.

Or, fin 2014 apparaissent les notions de projets et d'expériences. En effet, le Conseil régional de Champagne-Ardenne impose aux E2C des pédagogies "learning by doing ", soit des pédagogies par l'action, orientées vers la démarche expérientielle et les approches par projet. La coordination régionale E2C va les diffuser dans les écoles, alors même qu'elle ne s'était pas positionnée sur cette question jusqu'alors. Est alors négocié avec le financeur un démarrage lent d'une expérimentation sur deux projets pour l'établissement.

Ces pédagogies par projet sont réfléchies et expérimentées dans les dispositifs d'insertion des publics peu qualifiés, notamment à partir des années 1980 (Tiberghien-Bovy, op. cit.). Elles font explicitement référence aux théories développées par le philosophe pragmatique John Dewey (Dewey, 1938). Elles mettent en avant des apprentissages fondés sur des expériences progressives prenant sens dans la vie de la personne. Elles se réfèrent à l'approche par compétences (APC), qui affirme que l'expérience d'alternance 
est le support d'apprentissage par la réflexivité individuelle et collective : "Si l'accent est mis sur l'amélioration de compétences fonctionnelles et de connaissances pour mieux vivre au quotidien ou améliorer l'insertion socioprofessionnelle, les approches se centrent sur la question des compétences et des conditions de leur développement. " (Tiberghien-Bovy V., ibid., p. 33). Mais l'expérience d'alternance n'a d'intérêt que si elle devient support de réflexivité individuelle et collective, car toutes les situations, comme le rappelle Patrick Mayen (op. cit.), ne sont pas forcément apprenantes.

Cette orientation est lourde de conséquences, car elle pose la question des pré-requis des stagiaires, nécessaires ou non pour analyser une expérience. De fait, elle interroge les liens entre savoirs de base et activités-compétences et la manière dont les formateurs abordent cet accompagnement : "On s'aperçoit aussi que des compétences peuvent apparaître dans un contexte donné et disparaître si le contexte change. Il n'y a pas toujours corrélation entre le niveau strict de compétence à l'écrit et les pratiques» (Tiberghien-Bovy, ibid., p. 29). Il s'agit dès lors une réflexion de fond sur le contenu de leur activité professionnelle.

Ainsi, en 2016, l'établissement ardennais décide de revoir son approche d'accompagnement à l'orientation et à la gestion de projet dans son ensemble, et d'entrer pleinement dans une démarche compétences ${ }^{7}$. Elle va alors expérimenter la dynamique CléA, qui entraîne la délivrance d'un certificat par le Comité paritaire interprofessionnel national pour l'emploi et la formation (COPANEF), sur la base d'un référentiel. L'évolution vers le référentiel $\mathrm{Clé}^{8}{ }^{8}$ conduit tout individu à acquérir et/ou à faire valider les connaissances et compétences nécessaires à l'exercice d'une activité professionnelle. Ce dispositif délivre un premier diplôme à des personnes peu qualifiées et porte sur sept domaines :

1 - Communication en français ;

2 - Utilisation des règles de base de calcul et du raisonnement mathématique ;

3 - Utilisation des techniques usuelles de l'information et de la communication numérique ;

4 - Aptitude à travailler dans le cadre de règles définies d'un travail en équipe ;

5 - Aptitude à travailler en autonomie et à réaliser un objectif individuel ;

6 - Capacité d'apprendre à apprendre tout au long de la vie ;

7 - Maîtrise des gestes et postures et respect des règles d'hygiène, de sécurité et environnementales élémentaires.

7. Le laboratoire Lisec (Laboratoire interuniversitaire des sciences de l'éducation et de la communication), attaché à l'université de Lorraine, a été sollicité pour une recherche-action pour accompagner la mise en place de la démarche compétences pour l'E2C France, mais a pu être également sollicité par l'établissement ardennais.

8. La loi du 5 mars 2014 sur la formation professionnelle incite à la construction d'un référentiel CléA, fondé sur un socle de connaissances et de compétences professionnelles. 


\subsection{Travailler sur la construction de projet pour repenser la professionnalisation collective}

La démarche projet, mise en place depuis 18 mois, tend à se généraliser par un grand nombre d'expérimentations pédagogiques, - comme la construction d'une " mini entreprise " par exemple - avec des objectifs ciblés : "On se rend compte qu'il y a des jeunes qui se révèlent dans ce projet, ce que l'on n'aurait pas vu dans une démarche classique " (Formateur 10).

De plus, la thématique de la mobilité est au cœur des préoccupations de l'établissement depuis de nombreuses années. Mais au-delà d'un constat récurrent de mauvaises infrastructures de déplacement sur le territoire et des résistances des stagiaires, le travail sur la mobilité est considéré par la direction de l'établissement comme un levier pédagogique et de cohésion des trois pôles d'intervention autour de projets. Ce travail sur la base de la gestion de projets, pour les personnes en charge d'animer et d'accompagner la réflexion pédagogique dans l'établissement ardennais, a pour objectifs de favoriser la coordination des équipes et une professionnalisation collective.

Ces évolutions pédagogiques mettent en avant le fait que l'usage des outils doit certes améliorer l'accompagnement de l'individualisation des parcours des stagiaires, mais ne nécessite pas pour autant l'individualisation des modalités d'apprentissage. Cette évolution ne va pas de soi dans l'actuelle professionnalité des formateurs, centrée sur un suivi individualisé au détriment d'une démarche collective normalement induite par les projets : "Ma priorité, c'est de travailler avec le jeune et de l'amener à une certaine autonomie " (Formateur 11). L'analyse des entretiens et les observations sur sites ont mis en avant que cette approche individualisée reste prégnante parmi les formateurs. La transversalité ou la globalité des interventions sont peu formalisées dans les analyses du discours. Chaque formateur se recentre sur sa manière de travailler, en comparaison avec celle du collègue, et le projet reste une addition d'interventions : "Je travaille ma séquence de formation en fonction du niveau du jeune en mathématiques et de son besoin en stage " (Formateur 14). Cette relation particulière tissée avec le stagiaire est par ailleurs à mettre en lien avec la singularité du fonctionnement du "formateur référent " qui individualise le suivi.

L'évolution vers la démarche projet fait ressurgir, par ailleurs, la distinction entre formateurs et animateurs, que la répartition en trois pôles avait estompée. Les animateurs, qui jusque-là occupaient une place complémentaire par rapport au TRE et à la remise à niveau, sont maintenant au centre de la mise en ouvre de la démarche projet. C'est une évolution majeure de leur périmètre d'activité. Cependant, ils possèdent le niveau

9. Chaque stagiaire du dispositif E2C a un "formateur référent " qui supervise l'ensemble de son dossier et son suivi, tant lors de sa présence dans l'établissement qu'en période de stage, et à qui le stagiaire s'adresse en priorité durant la période passée à l'E2C. 
de diplôme le plus faible et les expériences les plus éloignées de la pédagogie. Les jeux de pouvoir, de reconnaissance et d'orchestration des savoirs sont ici remobilisés entre les acteurs (Terssac De, 1992).

L'analyse des observations et des discours révèlent que les regroupements, les réunions régulières de l'ensemble des formateurs, servent davantage à régler des problèmes administratifs ou d'organisation qu'à harmoniser les pédagogiques des trois pôles. Il apparait qu'au niveau de l'établissement, l'activité du « formateur référent », tout comme celle de l'« animateur projet » ne sont ni discutées collectivement, ni ne font l'objet d'analyse de pratiques afin de définir une professionnalité de l'accompagnement et surtout le périmètre de l'activité de chacun. Cet élément demeure, pour le moment, un point aveugle de l'animation du dispositif pour l'établissement.

\subsection{Les modes d'évaluation ne reconnaissent pas la professionnalité des formateurs}

Une réflexion s'opère progressivement de manière collective sur le statut de l'expérience et la manière d'en faire un réel objet de transfert, comme le préconise Patrick Mayen (2007). Cependant, actuellement, aucun travail structuré autour d'indicateurs sur l'évaluation ou l'analyse pédagogique n'est mené. Cela gêne les formateurs : "Ce qui nous manque aujourd'hui, c'est de l'analyse de pratiques, on a souvent tendance à incriminer le jeune " (Formateur 15). Les formateurs ont le sentiment d'être évalués davantage sur des performances de placement plus que sur leur pédagogie, ce qui génère une pression sur la finalité de leur travail : "Malheureusement, il n'y a pas d'évaluation sur la pédagogie, il n'y a que l'analyse des placements, c’est démobilisant pour les équipes".

La mise en place d'un suivi quantitatif sur les placements des bénéficiaires, par la coordination nationale, ajoutée à celle de l'établissement, apparaît comme de plus en plus pesante dans l'activité professionnelle des formateurs. Les objectifs et les résultats à atteindre sont subis comme du contrôle. Cette pression du chiffre est mal vécue par les formateurs : "Il a des raisons de chiffres, il y a des choses que je ne peux plus entendre, moi je suis sur l'humain" (Formateur 15). L'évaluation reste centrée sur les résultats, alors que l'on demande aux formateurs d'être dans un processus d'accompagnement induisant la réflexivité des stagiaires, les plaçant dans une injonction difficile à satisfaire (Santelman, 2015).

Les observations et les entretiens avec ceux qui coordonnent et animent l'ensemble du dispositif E2C de l'établissement ardennais révèlent que l'approche expérientielle ou par projet est saisie comme une opportunité d'innovation pédagogique devant porter le dispositif et ses formateurs. Cette évolution pédagogique, si elle amène une réflexion en interne, se trouve pour le moment face aux limites de sa mise en œuvre et de son animation. 


\section{Conclusion}

Lévolution en cours du dispositif E2C et des équipes de formateurs est liée à plusieurs niveaux et articulations du processus inachevé de professionnalisation. Cette professionnalisation concerne la redéfinition de l'organisation de l'activité, l'harmonisation des pratiques pédagogiques et l'offre de formation continue pour les formateurs. Mais cette recherche a permis de montrer que cette professionnalisation se réalise à deux échelles, au niveau régional et à celui de l'établissement. Nous avons montré comment l'établissement s'empare de la dynamique régionale pour pouvoir repenser son organisation, ses outils pédagogiques et la construction de ses équipes de formateurs.

La coordination comme l'établissement ardennais montrent, dans un premier niveau de professionnalisation, leur capacité à influencer le renouvellement des outils et des pratiques pédagogiques, à organiser de l'expérimentation. Ils favorisent ainsi la professionnalisation du dispositif par l'évolution de l'organisation du travail, un recrutement mieux ciblé, une offre importante de formation continue.

Mais un deuxième niveau de professionnalisation se lit dans l'harmonisation des techniques de recherche d'emploi (TRE), propre à l'établissement. Les réflexions en cours sont tributaires de l'accompagnement des équipes de formateurs, possédant des niveaux de formation et des expérience très disparates. Si des bilans et des outils sont bien repérés et permettent de passer d'une logique de travail sur autrui à une logique de travail avec autrui, cette approche du TRE reste fortement marquée par la relation du formateur avec le jeune.

Le troisième niveau de professionnalisation, en lien avec l'approche par projet et compétences, marque l'évolution de la professionnalité des formateurs, non plus centrée sur le suivi individualisé, mais appuyée sur des manières co-construites d'accompagner. L'établissement évolue certes vers ces pratiques pédagogiques ; cependant, les évaluations du dispositif se concentrent sur les indicateurs de placement, alors que, au moment de l'enquête, peu d'éléments qualitatifs sur les retours en formation sont proposés pour alimenter un accompagnement de pratiques professionnelles, l'expertise et la déontologie des formateurs.

\section{Bibliographie}

Aballéa F. (1992), «Sur la notion de professionnalité », Recherche sociale, n 124, pp. 39-49.

Astier I. (2009), "Les transformations de la relation d'aide dans l'intervention sociale ", Informations sociales, 152 , pp. 52-58.

Bardin L. (1977), L'analyse de contenu, Paris, Presses Universitaires de France, 291p. 
Blanchard S., Sontag J.-C. (2015), «Instaurer une relation de confiance pour accompagner les jeunes dans leur projet professionnel à l'école de la deuxième chance ", Éducation Permanente, numéro hors-série, pp. 137-146.

Bourdoncle R. (2000), "Autour des mots : professionnalisation, formes et dispositifs ", Recherche et Formation, 35, pp. 117-132.

Brun F., Corteel D., Pélisse J. (2012), « L’accompagnement des licenciés économiques dans le cadre du contrat de transition professionnelle : expertise et expérience ", Revue française de sociologie, Vol. 53, pp. 429-459.

Gasquet C. (2003), « Les jeunes sans qualification. Un groupe hétérogène, des parcours d'insertion divers ", Céreq Bref, 202, pp 1-4.

De Lescure E. (2005), «Le niveau des agents de la formation ne cesse de s'élever mais reste dispersé ", Éducation Permanente, n 165, pp. 39-50.

Demazière D. (2008), "L'ancien, l'établi, l'émergent et le nouveau : quelle dynamique des activités professionnelles ", Formation Emploi, n 101, pp. 41-54.

Demazière D. (2009), « Professionnalisation problématique et problématiques de la professionnalisation ", Formation Emploi, n 108, pp. 83-90.

Denechau B., Houdeville G., Mazaud C. (2015), À l'école de l'autonomie, épreuves et enjeux des dispositifs de la seconde Chance, Paris, L'Harmattan, $274 \mathrm{p}$.

Dewey J. (1938), Experience and education, New York, Collier, 91 p.

Dubar C. (2015), La formation professionnelle continue, Paris, La Découverte, Collection "Repères », 128 p.

Duvoux N. (2009), "L'injonction biographique dans les politiques sociales. Spécificité et exemplarité de l'insertion ", Informations sociales, n 156 , pp. 114-122.

Fourdrignier M. (2004), Transformations du rapport des jeunes au travail en Champagne Ardenne, les jeunes des dispositifs d'insertion, Rapport d'études pour la région ChampagneArdenne, 93 p.

Leclercq V. (2005), "La professionnalisation du formateur spécialisé en formation de base ", Éducation permanente, n 164, pp. 105-118.

Leclercq E. (2012), "Enseigner le Projet Professionnel, Personnalisé, une nouvelle approche pédagogique pour les enseignants chercheurs? ", Revue Carrefours de l'éducation, $\mathrm{n}^{\circ} 34$, pp. 161-175.

Legal L. (2015), "Les stages au cœur de la dynamique de formation », in Denechau B., Houdeville G., Mazaud C., A l'école de l'autonomie, épreuves et enjeux des dispositifs de la seconde Chance, Paris, L'Harmattan, 274 p. 
Mayen P. (2007), « Passer du principe d'alternance à l'usage de l'expérience en situation de travail comme moyen de formation et de professionnalisation ", in Merhan F. et al., Alternances en formation, Bruxelles, De Boeck Supérieur, "Raisons éducatives ", pp. 83-100.

Riot L. (2010), «Les pratiques des formateurs dans le cadre des dispositifs d'insertion : le projet en théorie, les compromis en pratique ", in de Lescure E. \& Frétigné C. (Dir), Les métiers de la formation. Approches sociologiques, Rennes, PUR, 236 p.

Rogers C. (1968), Le développement de la personne, Paris, Dunod, 274p.

Santelman P. (2015), «Introduction », in Quelle pratiques pédagogiques dans l'accompagnement des publics peu qualifiés, numéro hors-série 7, Éducation permanente.

Terssac de G. (1992), Autonomie dans le travail, Paris, Presses universitaire de France, $279 \mathrm{p}$.

Tiberghien-Bovy V. (2007), "La formation de base : publics, dispositifs pratiques ", Savoirs, n²14, pp. 8-55.

Winnicott D. (1975), Jeu et réalité, l'espace potentiel, Paris, Gallimard, 288 p.

Wittorski R. (2007), Professionnalisation et développement professionnel, Paris. L'Harmattan. 